tein into whatever different structure was the most easily attainable, they specifically set out to convert the B1 domain of protein G, which consists of a four-stranded $\beta$-sheet packed against a single helix ${ }^{4}$, into a new protein with the folded structure of another protein, Rop (repressor of primer). Rop is an RNA-binding protein that folds as two helices associating to form a four-helix bundle ${ }^{5}$. Their strategy was to identify the key residues of the B1 domain that encode its mostly $\beta$-sheet structure, and to replace those residues with the corresponding, structurally critical amino acids of Rop.

What are the essential features of a protein that we have to specify to define its fold? There are four important goals, all of which aspiring protein designers must achieve ${ }^{6}$. The first is to initiate and terminate elements of secondary structure (the helices and $\beta$-sheets that are shown as ribbons in structure cartoons; Fig. 1). The second is to create a hydrophobic core - a congregation of water-avoiding amino-acid side chains whose reluctance to be exposed to the solvent will stabilize the correctly folded structure. The third goal is to supply sequences that encode loops and hairpin turns in the protein backbone, to connect sequential, secondary-structural elements. Finally, specific tertiary links need to be created; that is, energetically favourable interactions between amino acids that are distant in the sequence.

Following this logic, to swap the protein identities Regan and co-workers first had to replace residues that had an empirically observed tendency to form sheets, with other residues that prefer to be in a helix. Then they constructed the appropriate core structure based on their previous characterization of simplified core variants of Rop ${ }^{7,8}$. They also pinched from the Rop sequence a salt bridge (a pair of oppositely charged amino acids) and a single tyrosine residue as a spectroscopic probe of the folded state.

These modifications account for 28 amino acids - exactly $50 \%$ of the original B1 sequence. As well as the 56 residues that form the helices (and were the design target in this study), Rop has an unstructured 'tail' of seven residues. This was found to be essential for the good water solubility of Rop, although not for its structure, and it was also included in the design.

The new protein was fittingly called Janus (Fig. 1), as it looks like the B1 domain on the sequence level but was expected to show the face of Rop on the structural level. Regan's group cloned Janus into bacteria so they had enough material for structural investigations and could address the crucial question does Janus really look both ways? As they had predicted, Janus showed the circular dichroism and nuclear magnetic resonance signatures, isotope-exchange properties and highly cooperative unfolding that would be expected of a folded protein with the structure of Rop.
Based on these results, it was agreed that Janus had met the criteria of the challenge, and Regan was awarded the Paracelsus prize in a small ceremony last month.

Although this achievement is an impressive demonstration of just how far our knowledge of the determinants of protein structure has come, a few questions remain. For example, can the limit of sequence identity be pushed higher than $50 \%$, and can the transformation be done in the opposite direction? Regan's group is now addressing the limits of the sequence identity by reverting the changes made from B1 to Janus, one by one. But it might be more difficult to do the transformation in the opposite direction, as the formation of $\beta$-sheet structures depends on interactions between sites that may be far apart in the sequence. Still, the demonstration that protein designers now know which half of the text they have to rewrite to change a protein fold means that, one day, we will be able to read and write the code of protein folding.

Michael Groß is at the Oxford Centre for Molecular Sciences, Oxford OX1 3QT, UK. Kevin W. Plaxco is in the Department of Biochemistry, University of Washington, Seattle, Washington 98195, USA.

1. Rose, G. D. \& Creamer, T. P. Proteins Struct. Funct. Genet. 19, 1-3 (1994).

2. Dalal, S., Balasubramanian, S. \& Regan, L. Nature Struct. Biol. 4, 548-552 (1997).

3. Chothia, C. \& Lesk, A. M. EMBO J. 5, 823-826 (1986).

4. Gronenborn, A. M. et al. Science 253, 657-661 (1991).

5. Banner, D. W., Kokkinidis, M. \& Tsernoglou, D. J. Mol. Biol. 196, 657-675 (1987).

6. Cordes, M. H. J., Davidson, A. R. \& Sauer, R. T. Curr. Opin. Struct. Biol. 6, 3-10 (1996).

7. Munson, M., O'Brien, R., Sturtevant, J. M. \& Regan, L. Prot. Sci. 3, 2015-2022 (1994).

8. Munson, M. et al. Prot. Sci. 5, 1584-1593 (1996).

\title{
Sociology of science
}

\section{Irregular scientific conjugations}

The perception of behaviour varies with the distance of the observer from the subject. This variation generally extends along a continuum but, in the special case of the behaviour of scientists, three primary clusters of distance seem particularly important. These can be defined as identity (I), unrelatedness $(U)$ and separated/heterologous (S/he). Results in ten areas of behavioural perception within science can thus be summarized as irregularly conjugating verbs.

I construct precise and logical sentences You write in a standard scientific style She produces incomprehensible papers riddled with jargon

I am frequently asked to appear in TV science documentaries

You are a typical eccentric media-friendly boffin

He is a self-promoting pseudoscientific media whore

\section{I am a polymath}

You are a jack of all trades

She has views on subjects she knows

nothing about

I publish every finding for the wider scientific community

You have a refined strategy for

building an impressive publications list

They are salami slicing

I am a confident speaker in panel discussions You are charmed by the sound of your own voice

He shouts down the views of other participants

I have a reputation that enables me to attract bright young scientific talent You have a reputation maintained by a highly able team of collaborators He has a reputation for exploiting poorly paid postdocs
I ensure that a company's scientific approach is up to date

You reassure investors by your presence on the Scientific Advisory

Board

He receives a fat retainer simply because he has a Nobel prize

I undertake peer-review carefully and thoroughly

You delay returning your comments on the paper

She conducts additional experiments for her own paper while the manuscript languishes in her pending tray

I do not publish preliminary data You conduct follow-up experiments in order to maintain a scientific lead over competitors

He hoards data until his industrial collaborators have filed patents

I actively solicit and review high-quality papers

You provide useful comments on the occasional manuscript

They are only on the editorial board to preserve the façade of geographical balance

\section{John Hodgson}

John Hodgson is a senior editor of Nature Biotechnology. 